\title{
Análisis de la Inversión en Investigación y Desarrollo en el Panorama Actual de México
}

\author{
Diego Saldaña-Ulloa ${ }^{a}$, Amanda Martínez-Pedraza
}

\begin{abstract}
In this work We analyze the actual situation of Mexico about the investment in Research and Development. We use actual data to give a better context and we identify several problems that must be resolved. The main topics that we analyzed are focused on employment in Research and Development, the brain drain effect, the situation about the patents in Mexico and the analysis of maquiladora-industry and how is related with Research and Development investment.
\end{abstract}

\section{INTRODUCCIÓN}

El Producto Interno Bruto (PIB) nos permite conocer el grado de actividad económica de un país ó región. Habitualmente este indicador macroeconómico está asociado con la capacidad de producción de bienes y servicios. Un mayor PIB siempre está relacionado con regiones ecónomicas fuertes por lo que a traves de los años se ha adoptado como el principal factor a tener en cuenta para la clasificación de una economía. Por esta misma razón, el PIB se ha utlizado como una medida del desarrollo económico [16]. Si bien no podemos afirmar que el PIB sea sinónimo de desarrollo a nivel general (ya que la medida de desarrollo de una región abarca multiples facetas que no solo se relacionan con el tema económico), nos da un buen indicio del comportamiento de un país o región.

Por otro lado, cierto porcentaje del PIB anual es destinado por los gobiernos para el gasto en diferentes áreas (pago de deuda, gasto público, inversión en investigación y desarrollo, etc) lo que se traduce en un aumento o disminución de la actividad económica de una región [10]. Muchos paises desarrollados destinan un porcentaje significativo del PIB (más del 2\%) en investigación y desarrollo tecnológico [4]. Es por ello que la inversión en Investigación y Desarrollo Tecnológico es sinónimo de un aumento en la actividad económica y mejora de la calidad de vida de la población.

En este trabajo abordaremos la problemática de México frente a su baja inversión en Inverstigación y Desarrollo tomando en cuenta el papel del gobierno y el sector privado.

November 2019

${ }^{a}$ E-Mail: diego.ulloa13@hotmail.com

\section{El Problema de la InVERsión en InVEStigación y Desarrollo en MÉXico}

El desarrollo económico ha venido de la mano de la inversión en Investigación y Desarrollo (ID). Los paises desarrollados tienden a gastar más del $2 \%$ de su PIB en este rubro. Podemos encontrar una basta literatura al respecto, sobre el análisis o correlación entre la inversión en ID y el desarrollo económico de un país [23] [14] [19]. Es por ello que actualmente en el caso particular de México, muchos sectores dentro del área de la investigación, exigen un aumento en el presupuesto en materia de Ciencia y Tecnología.

Un aumento en la inversión en ID siempre traerá consigo desarrollo a corto, mediano o largo plazo. En paises desarrollados, la inversión en ID se centra en el desarrollo de nuevas tecnologías mientras que en paises en vías de desarrollo la inversión se centra en importación de tecnologías extranjeras [8]. De la misma forma se tiene que hacer una distinción entre la inversión en ID pública y privada ya que son rubros diferentes pero que tienden a complementarse. En el caso de México, por ser considerado un pais en vías de desarrollo, la mayor aportación en ID se da por parte del gobierno. Esto se encuentra en común acuerdo con literatura al respecto sobre paises en vías de desarrollo y su relación con la inversión en ID [8]. Para que este sistema pueda coexistir (y posteriormente esperar un crecimiento económico), se necesita de una participación de ambas partes (pública y privada) mediante acuerdos que permitan el flujo y desarrollo del conocimiento para el desarrollo de proyectos [5]. En el panorama actual de México, esto supone un gran obstaculo a vencer ya que se tiene que convencer a la iniciativa privada principalmente de dos cosas: 1) invertir más en ID (para el desarrollo de sus productos ó servicios) y 2) establecer más vinculos de participación en proyectos conjuntos. Las principales áreas de inversión en ID para las empresas privadas (en México) se encuentran en las industrias petroquímicas, minería y construcción y alimentos. A pesar de ello no se puede comparar con la inversión en ID que realizan (a nivel internacional) otras empresas extranjeras [17].

La implementación de políticas públicas adecuadas que permitan un participación en ID entre el sector público y privado es uno de los principales retos en la economía actual 
de México. Si la inversión solo proviene de un sector (por ejemplo en la situación actual, el sector público), pueden existir efectos que no necesariamente se traducirían en un desarrollo económico. Por ejemplo, la falta de empleo en el área de investigación, fuga de cerebros, bajo o nulo desarrollo de patentes. Estos puntos serán tratados en las secciones siguientes.

\section{EMPLEABILIDAD EN EL ÁrEA DE ID}

Como ya hemos comentado, existe una extensa literatura y evidencia sobre la inversión en ID y su impacto en el crecimiento económico de un país. Es por ello que no resulta ilógico, que durante esta decada, el $32 \%$ de los alumnos de nuevo ingreso en educación superior corresponden a areas relacionadas con la Ciencia, Tecnología, Ingeniería y Matemáticas (STEM). Esto convierte a México en uno de los paises con mayor proporción de estudiantes dentro de las STEM entre los paises miembros de la OCDE [20].

El porcentaje de afinidad laboral dentro de las STEM se mantiene en alrededor del 20\% [18]. Adicionalmente, los sectores de mayor ocupación (dentro de estas areas) son: educación e investigación, servicios gubernamentales y comercio al por menor [22]. A pesar de estas cifras, no existe un indicador que nos proporcione información sobre el porcentaje de empleabilidad en el sector público y privado. Es importante tomar en cuenta esta cifra, ya que en los paises desarrollados el 70\% de graduados en STEM trabajan en el sector privado, mientras que el 30\% restante en educación y gobierno [11]. Esta correlación entre investigación pública y privada y su impacto en el desarrollo económico, ha sido previamente estudiada [5]. La creación de políticas públicas mediante las cuales se incentive a ambos rubros, es plenamente necesaria.

\section{Fuga de Cerebros}

Durante los ultimos 20 años, aproximadamente 1357000 profesionistas han dejado el pais [1], poco mas de un millon se encuentran en Estados Unidos. Esta fuga del conocimiento es un tema delicado de tratar ya que estamos ante dos situaciones de problematica mutua: 1) La inversión en formación de recursos humanos en ID y 2) la pérdida del dinero invertido en la formación de esos recursos humanos (para el país). Hasta la decada pasada, la inversión en la formación de investigadores competentes tuvo un costo de poco más de 1140 millones de pesos (tomando en cuenta el indice de precios e inflacion de hace 10 años) [24]. Actualmente, de acuerdo con datos de la actual directora del Consejo Nacional de Ciencia y Tecnología (CONACYT), durante el último sexenio se registró que unos 30 mil profesionistas con formación de posgrado, se encuentran en el extranjero debido a la falta de oportunidades laborales dentro de México.
Adicionalmente, los datos anteriores no toman en cuenta la proporcion de graduados sin posgrado, que por los mismos motivos (y la brecha salarial), deciden migrar laboralmente. De acuerdo con el Diario EL PAIS, México es el lider de los paises latinoamericanos en fuga de cerebros [9]. Esto supone un gran problema para México ya que tan solo hasta 2017 existían 54420 becas (en curso) por lo que existirán alrededor de 50000 nuevos profesionistas de posgrado en el corto plazo.

Existen trabajos que estudian los efectos de la fuga de cerebros en economías en vías de desarollo [6]. Entre los resultados que se pueden observar, es que hay un beneficio para los paises (en términos de mejoras en la educación) que permiten una migración laboral. Sin embargo efectos adversos pueden desarrollarse si el porcentaje de fuga de cerebros es alto o existen grandes diferencias salariales respecto del país al que migran los profesionistas.

\section{El Tema de las Patentes}

Para que una región ó país tenga desarrollo económico al invertir en ID, es necesario que los resultados (tangibles) de las investigaciones (que lo ameriten) seán registrados mediante patentes. Esto garantiza que exista un flujo ecónomico sobre el uso de la tecnología (inovación) en el país de registro. El uso de las patentes permite la creación de nuevos productos con capacidad comercial que pueden ser explotadas por empresas (nuevas o consolidadas) y el gobierno.

Existen trabajos previos sobre el impacto positivo entre el desarrollo de patentes y el crecimiento económico [12] [23]. En el caso de Mexico, el TLCAN ha permitido que exista un crecimiento económico mediante el registro de patentes no residentes [12]. Sin embargo, como se ha mencionado en un apartado anterior, esto genera una dependencia de tecnología extranjera, lo cual no necesariamente es positivo para un país en vías de desarrollo como México.

Estudios sobre el tema han sido publicados evidenciando el dominio sobre el registro de patentes extranjeras (no residentes) en México [12]. Si nos permitimos hacer una comparación con paises desarrollados, en ellos existe un balance entre el número de patentes residentes y no residentes, lo cual no es el caso de México. De acuerdo con información de WIPO en 2018 habían 8464 patentes no residentes otorgadas y tan solo 457 patentes residentes otorgadas [21]. Dentro de este apartado es conveniente mencionar el Tratado de Cooperación en Materia de Patentes (PCT) que permite la protección de una patente entre los paises miembros. En el caso de México los lideres del PCT corresponden a Centros de Investigación Públicos, mientras que en el caso de nuestro vecino del norte (USA, pais desarrolado) los lideres corresponden a empresas (privadas) de su territorio [21]. Las 
patentes registradas con PCT garantizan una competencia económica internacional.

Las patentes residentes solicitadas y otorgadas por miembros de Instituciones de Investigación Pública, ameritan su propio análisis por la poca proporción que representan respecto de las patentes no residentes. Alenka et al ha identificado algunas caracterisiticas sobre la problemática de la baja tasa de patentes registradas y otorgadas por miembros del SNI [13]. Entre las causas que se logran citar se incluyen la edad, el apoyo de las instituciones y el número de investigadores (a nivel nacional). Es indiscutible que póliticas públicas para incentivar el desarrollo y registro de patentes (de actores públicos y privados) deben llevarse a cabo, para en esa medida, aumentar el desarrollo económico del país.

\section{MÉXICO Y LA MAQUILACIÓN}

Practicamente desde los años 90, México ha sido considerado un país Maquilador. Este fenómeno se acuño debido a los acuerdos surgidos del TLCAN [7] en términos de importación y exportación de productos. Grandes empresas transnacionales tienen un modelo de negocio rentable basado en este sistema de producción (mano de obra barata). Si bien el término maquiladora se refiere a empresas que están excentas de pago de aranceles por importación de materias primas, al día de hoy se utiliza como sinónimo del proceso industrial que consiste en el ensamblaje o producción en serie de bienes. El modelo maquilador supone un buen incentivo para trabajadores y empresarios, en primer lugar por el desarrollo económico que se da en una región (creación de fuentes de empleo) y en segundo lugar debido a que la inversión se ve reflejada en la producción.

Sin embargo, este modelo no resulta rentable para un país cuya inversión en ID es baja, ya que como lo hemos apuntado en secciones anteriores, la inversión en ID siempre traerá consigo un aumento del crecimiento económico. Esto se refleja en el crecimiento modesto que ha tenido la economía Mexicana desde la decada de los 90 (a pesar de la puesta en marcha del TLCAN) [15].

Ya que el modelo de maquilación no toma en cuenta la inversión en ID, resulta cuestionable si la implementación de este tipo de producción influye en la dinámica de la creación de nuevas empresas en el país (es decir, empresas sólo interesadas en producción y no en ID). Con el desarrollo de la era digital, una parte del modelo maquilador ha evolucionado a la creación de empresas de outsorcing. Mediante la subcontratación (outsourcing) empresas nacionales y extranjeras pueden hacer uso de recursos humanos (altamente calificados) para dar solución a sus necesidades.

Parte del outsourcing que podemos encontrar en la actualidad corresponden a empresas tecnológicas que hacen uso de personas del área de las STEM. A pesar de ello hay que aclarar que el modelo de outsourcing no es sinónimo de inversión en ID. Podríamos decir que hasta ahora el papel del país no ha sido de Inovación y Desarrollo, si no más bien de producción en serie.

En este mismo sentido podemos hacer una analogía entre el fenómeno maquilador y la producción científica del país. Como ya hemos mencionado, México tiene el mayor porcentaje de estudiantes de educación superior que ingresan a una carrera relacionada con las STEM. Por lo que la perspectiva a futuro a punta al aumento de recursos humanos en esta área. De igual forma, el país ha tenido un aumento (más del doble) en su producción científica (artículos) durante los últimos 15 años. De acuerdo datos del Banco Mundial [2] en 2003 se publicaban alrededor de 6600 artículos del área de las STEM mientras que en 2016 esa cifra aumentó a 14 500. Ha pesar de esto, durante el mismo periodo no hubo un aumento significativo en el PIB [3]. En este sentido, México se ha especializado en aumentar su producción científica sin que ello signifique un impacto en su crecimiento económico, esto en una especie de "maquiladora científica" para el país.

\section{CONCLUSIONES}

En este trabajo se realizó un análisis amplio del panórama actual de la inversión en ID en México. Existe evidencia significativa de que la inversión en este sector impulsa en el corto, mediano y largo plazo el crecimiento económico de un país, ya sea en vías de desarrollo o paises desarrollados. Esta inversión debe provenir tanto del sector público como del privado en una especie de coexistencia mutua. Como lo hemos mencionado, la mayor parte de la inversión en ID en México se realiza por parte de la administración pública. Una baja inversión en ID por parte del gobierno, así como el poco interés de la iniciativa privada en ID, son una de las causas del bajo crecimiento económico de Mexico durante los ultimos 20 años. De acuerdo con estadísticas de la OCDE se prevee que el país tenga un aumento en la formación de recursos humanos dentro de las STEM. A pesar de existe un porcentaje razonable de afinidad laboral para este sector, sin una correcta implementación de políticas públicas que incentiven la inversión en ID se corre el riesgo de un aumento del porcentaje de fuga de cerebros (perdida económica para el país).

Adicionalmente, la inversión en ID debe venir acompañada del desarrollo de patentes para que de esta forma exista un flujo económico. Actualmente, existe un mayor número de patentes no residentes en comparación de las residentes. En paises desarrollados este número tienden a un equilibrio. Mas aún los lideres en solicitud de patentes PCT corresponden a la iniciativa privada (residentes) mientras que en México los lideres corresponden al sector público.

Finalmente, el concepto maquilador aún sigue presente en el país. Este tipo de modelo ha sido implementado en otros sectores como el tecnológico (outsourcing) lo cual nos pone 
en una desventaja respecto de la competencia económica internacional. Dentro del fenomeno de maquilación hemos identificado una analogía con el aumento de la producción científica de México que no necesariamente se ha traducido en un crecimiento ecónomico. Esta especie de "maquiladora cientifica" puede tener diferentes causas como la baja inversión en ID del sector público y privado y el poco interés de las empresas. Hay que enfatizar que ningún modelo basado en la maquilación es sinónimo de inversión en ID. La inversión en ID generalmente se traduce en la creación de empresas, desarrollo de patentes y un posterior crecimiento económico, esto no corresponde al caso de México. Sólo mediante una correcta alianza entre gobierno y empresas se pueden resolver los conflictos que se siguen teniendo respecto de la inversión en ID y el posterior crecimiento económico del país.

\section{REFERENCES}

[1] J. Avila. La migración internacional calificada: tendencias y lecciones del movimiento de mexicanos a estados unidos. México, ponencia presentada (en representación del Conapo) en la $95^{a}$ Jornada Informativa del ime: Vínculos con la Diáspora Calificada, la Red de Talentos Mexicanos en el Exterior, 2012.

[2] The World Bank. Scientific and technical journal articles - mexico. National Science Foundation, Science and Engineering Indicators., 2016.

[3] The World Bank. Crecimiento del pib ( Datos sobre las cuentas nacionales del Banco Mundial y archivos de datos sobre cuentas nacionales de la OCDE., 2018.

[4] World Bank. Research and development expenditure (The World Bank, pages https://data.worldbank.org/topic/science-and-technology, 2017.

[5] B. Becker. Public $r \& d$ policies and private $r \& d$ investment: A survey of the empirical evidence. Journal of Economic Surveys, pages 917942 doi: 10.1111/joes.12074, 2014.

[6] M. Beine, F. Doquier, and H. Rapoport. Brain drain and economic growth: theory and evidence. Journal of Development Economics, 64:275-289, 2001.

[7] L. Bendesky, E. Garza, J. Melgoza, and C. Salas. La industria maquiladora de exportación en méxico: mitos, realidades y crisis. Estudios Sociológicos, 22:283-314, 2004.

[8] V. Bilas, M. Bosnjak, and T. Cizmic. Relationship between research and development and economic growth in the eu countries. 13th International Scientific Conference on Economic and Social Development, pages 223-230, 2016.

[9] R. Colleta. México, campeón latinoamericano de fuga de cerebros. El País, 2018.

[10] P. Reyes M. Díaz, M. Mejía and A. Desiderio. Efectos del gasto público en el pib en los estados de méxico, 1999-2014. Investigación Económica, 77(305):74-96. https://dx.doi.org/10.22201/fe.01851667p.2018.305.67484, 2018.

[11] National Science Foundation. S\&e workers in the economy. NSF, 2018.

[12] A. Guzman, H. Gomez, and F. Lopez. Patents and economic growth, the case of mexico during the nafta. Economía Teoría y Práctica, pages 177-2014, 2018.

[13] A. Guzman, N. Molina, and G. Calderon. Factors affecting the propensity of academic researchers in mexico to become inventors and their productivity. Contaduría y Administración, 64, 2018.

[14] INCyTU. Inversión para ciencia, tecnología e innovación en méxico. FCCyT, 011, 2018.

[15] T. Kehoe and F. Meza. Crecimiento rápido seguido de estancamiento: México (1950-2010). EL TRIMESTRE ECONÓMICO, LXXX:237280, 2013.
[16] M. Kummar and A. Castro. Is gdp an adequate measure of development? International Growth Centre, pages https://www.theigc.org/blog/is-gdp-an-adequate-measure-ofdevelopment/, 2019.

[17] J. Lopez. Seis firmas mexicanas que sí invierten en innovación. El Financiero, 2015.

[18] Observatorio Laboral Mexico. Tendencias del empleo profesional segundo trimestre 2019. Observatorio Laboral 2019, 2019.

[19] J. Nkwoma. The contribution of $r \& d$ expenditure to economic growth in developing economies. Social Indicators Research, 124(3), pages 727-745 https://doi.org/10.1007/s11205-014-0807-3, 2014.

[20] OCDE. Panorama de la educación 2017. OCDE, 2017.

[21] World Intelectual Property Organization. Statistical country profile. WIPO, 2018.

[22] Instituto Mexicano para la Competitividad A.C. Compara carreras 2018. Instituto Mexicano para la Competitividad A.C., 2018.

[23] P. Pegkas, C. Staikouras, and C Tsamadias. Does research and development expenditure impact innovation? evidence from the european union countries. Journal of Policy Modeling, page https://doi.org/10.1016/j.jpolmod.2019.07.001, 2019.

[24] C. Tigau. Riesgos de la fuga de cerebros en mÉxico: Construcción mediÁtica, posturas gubernamentales y expectativas de los migrantes. Centro de Investigaciones sobre América del Norte México, 2013. 